\title{
Logarithmically enhanced Euler-Heisenberg Lagrangian contribution to the electron gyromagnetic factor
}

\author{
Andrzej Czarnecki $\odot,{ }^{1}$ Jan Piclum, ${ }^{2}$ and Robert Szafron $\oplus^{3, *}$ \\ ${ }^{1}$ Department of Physics, University of Alberta, Edmonton, Alberta, Canada T6G $2 E 1$ \\ ${ }^{2}$ Theoretische Physik 1, Naturwissenschaftlich-Technische Fakultät, Universität Siegen, 57068 Siegen, Germany \\ ${ }^{3}$ Theoretical Physics Department, CERN, 1211 Geneva 23, Switzerland
}

(Received 20 August 2020; accepted 29 October 2020; published 11 November 2020)

\begin{abstract}
Contrary to what was previously believed, two-loop radiative corrections to the $g$ factor of an electron bound in a hydrogenlike ion at $O\left(\alpha^{2}(Z \alpha)^{5}\right)$ exhibit logarithmic enhancement. This previously unknown contribution is due to a long-distance light-by-light scattering amplitude. Taking an effective field theory approach, and using the Euler-Heisenberg Lagrangian, we find $\Delta g=-\left(\frac{\alpha}{\pi}\right)^{2}(Z \alpha)^{5} \frac{56 \pi}{135} \ln Z \alpha$.
\end{abstract}

DOI: 10.1103/PhysRevA.102.050801

The gyromagnetic factor $g$ describes the proportionality of a particle's magnetic moment $\boldsymbol{\mu}$ to its spin $\boldsymbol{s}$,

$$
\boldsymbol{\mu}=g \frac{e}{2 m} \boldsymbol{s},
$$

where $e$ is the particle's charge, $m$ its mass, and we use units $c=\hbar=1$. Boldface letters denote usual vectors.

We consider the electron, for which Dirac's theory predicts $g=2$. This value is corrected at the per mille level by the electron's self-interactions, at present known to the fifth order in the fine-structure constant $\alpha \simeq 1 / 137[1,2]$.

Larger corrections can arise from the electron's interaction with its environment. The simplest such influence is the Coulomb field of the nucleus to which the electron is bound. These binding corrections are reviewed in Ref. [3]. Full numerical evaluation of two-loop self-energy diagrams is under way [4] and first results for a class of diagrams are already available [5,6].

These calculations are of great metrological interest, because the electron mass [ $m$ in Eq. (1)] is best determined with an ion in a Penning trap, rather than by trapping an electron alone (binding to a nucleus greatly decreases errors caused by the electron's thermal motion). In the future, a competitive value of the fine-structure constant may also be obtained from such measurements [7-9], complementary to atom interference [10] and the free-electron $g-2$ [11]. The potential of the bound $g$ factor to constrain scenarios beyond the standard model is discussed in Ref. [12].

Neglecting nuclear structure corrections, $g$ for the $1 s$ state can be expressed in a double series in powers of $\alpha / \pi$ (selfinteractions) and in powers and logarithms of $Z \alpha$ (interactions

\footnotetext{
*robert.szafron@cern.ch

Published by the American Physical Society under the terms of the Creative Commons Attribution 4.0 International license. Further distribution of this work must maintain attribution to the author(s) and the published article's title, journal citation, and DOI.
}

with the nucleus). With $L=-\ln (Z \alpha)^{2}$,

$$
\begin{aligned}
g= & \frac{2}{3}\left[1+2 \sqrt{1-(Z \alpha)^{2}}\right]+\frac{\alpha}{\pi} \sum_{i, j=0}^{\infty} a_{i j}(Z \alpha)^{i} L^{j} \\
& +\left(\frac{\alpha}{\pi}\right)^{2} \sum_{i, j=0}^{\infty} b_{i j}(Z \alpha)^{i} L^{j}+O\left(\left(\frac{\alpha}{\pi}\right)^{3}\right) .
\end{aligned}
$$

This structure mirrors the expansion of atomic energy levels (Lamb shift [13]) and so far it has been found that if a logarithm is present in one observable in a given order, it is also present in the other. This rule is very important because the Lamb shift is better understood theoretically than the $g$ factor. Measurements with ions of various $Z$ have been used to fit unknown coefficients in Eq. (2) [3] to extract the electron mass.

Here we find the first exception to this rule: in the Lamb shift the coefficient corresponding to $b_{51}$ vanishes, whereas we find that

$$
b_{51}=\frac{28 \pi}{135} .
$$

In principle, logarithmic effects can always be calculated in at least two ways. The argument $Z \alpha$ is really a ratio of two distance scales, for example, the large Bohr radius and the small electron Compton wavelength. One can calculate only the long-distance or short-distance part. In both cases one finds the same magnitude of logarithmic divergence.

In the present case, we did both, to be sure that the logarithmic contribution really exists. Below we briefly outline both parts of the calculation. We leave for future work the evaluation of the nonlogarithmic part, together with providing further technical details of the computation.

The new logarithmic contribution is an effect of the virtual light-by-light (LBL) scattering. It arises through the coupling of four photons induced by their interaction with a virtual charged particle such as an electron. Figure 1 provides an example, where the Coulomb field of the nucleus couples to an external magnetic field, and two photons are interacting with 


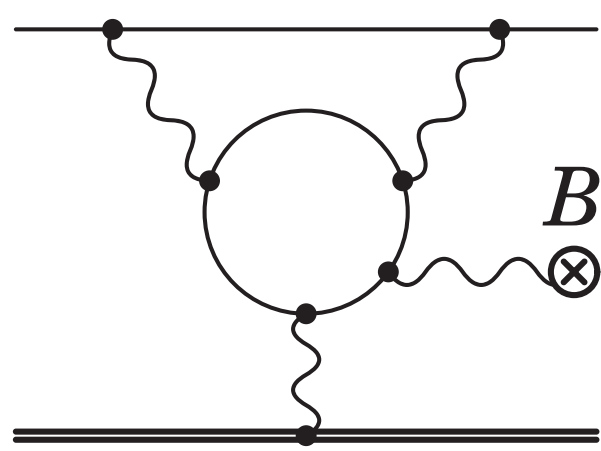

FIG. 1. An LBL loop contributing to $\alpha^{2}$ corrections to the bound electron $g$ factor. The thick double line denotes the nucleus. Thin solid lines are electrons and wavy lines are photons, including the external magnetic field $\boldsymbol{B}$.

the bound electron, thus changing the electron's $g$ factor and modifying its response to the magnetic field.

LBL was first predicted by Heisenberg and Euler [14,15] and by Weisskopf [16] who determined corrections to Maxwell's Lagrangian of the electromagnetic field $\mathcal{L}=$ $\frac{1}{2}\left(\boldsymbol{E}^{2}-\boldsymbol{B}^{2}\right)$,

$$
\mathcal{L}_{\mathrm{EH}}=\frac{\alpha^{2}}{m^{4}}\left[c_{1}\left(\boldsymbol{E}^{2}-\boldsymbol{B}^{2}\right)^{2}+c_{2}(\boldsymbol{E} \cdot \boldsymbol{B})^{2}\right],
$$

with $c_{1}=\frac{2}{45}$ and $c_{2}=\frac{14}{45}$. While $\mathcal{L}$ leads to linear Maxwell's equations, $\mathcal{L}_{\text {EH }}$ introduces nonlinear effects. Nowadays, this classic result is often the first nontrivial example encountered by students learning effective field theory methods.

Searches for effects of $\mathcal{L}_{\mathrm{EH}}$ have so far been in vain [17]. Observed nonlinear effects arise either from interactions with matter (nonlinear optics) or from high-energy processes with photon momenta much larger than the electron mass, beyond the validity of $\mathcal{L}_{\mathrm{EH}}$. For example, photon splitting $\gamma N \rightarrow$ $\gamma \gamma N$ has been measured [18] (see [19] for a theoretical review). A related process is Delbrück scattering $\gamma N \rightarrow \gamma N$ [20]. The high-energy LBL scattering has been observed in ultraperipheral heavy-ion collisions [21].

The $\mathcal{L}_{\text {EH }}$ effect described in this Rapid Communication likely has the best chance of being experimentally accessible.

It often happens with bound-state radiative corrections that a single Feynman diagram contributes to different orders in the perturbative expansion. To disentangle corrections of different orders, it is convenient to use the expansion by regions [22-26]. Once the relevant modes are identified, a systematic expansion can be achieved by setting up an effective field theory (EFT) whose operators capture the low-energy physics, while the so-called matching coefficients contain information about short-distance phenomena.

In bound-state quantum electrodynamics (QED), the relevant EFT is obtained in a two-step process. First, we integrate out the hard modes, i.e., momenta of the order of electron mass $m$. The resulting theory is known as nonrelativistic QED (NRQED), introduced by Caswell and Lepage [27]. The NRQED Lagrangian is organized in powers of the electron's velocity (in an ion with the atomic number $Z$, that velocity is $v \sim Z \alpha$ ), or inverse powers of electron mass [28]. The Euler-
Heisenberg $(\mathrm{E}-\mathrm{H})$ Lagrangian $\mathcal{L}_{\mathrm{EH}}$ is part of the NRQED Lagrangian and contributes at $O\left(v^{4}\right)$.

NRQED is still complicated and contains modes with a range of energy scales. In the second step, one integrates out soft modes whose momenta scale as $m v$, and potential photons with energy $E \sim m v^{2}$ and three-momentum $\boldsymbol{p} \sim m v$. The resulting theory is called potential NRQED (PNRQED) [29-31]. It contains instantaneous, nonlocal interactions between the electron and the nucleus, the so-called potentials.

The leading one is the Coulomb potential responsible for the binding and described by the operator

$$
\int d^{3} r\left[\chi_{e}^{\dagger} \chi_{e}\right](\boldsymbol{x}+\boldsymbol{r})\left(-\frac{Z \alpha}{r}\right)\left[N^{\dagger} N\right](\boldsymbol{x}),
$$

where $\chi_{e}$ is the nonrelativistic electron field, and $N$ is the nucleus field. Other potentials are treated as perturbations.

To compute the contribution of the E-H interaction to the bound electron $g$ factor, we have to generalize potentials to include spin-dependent interactions with an external magnetic field. The two-step EFT approach has been successfully used to compute spin-independent observables before. Technical details can be found in Ref. [32] (see also [33-38]).

LBL scattering first contributes to the bound electron $g$ factor at $O\left(\alpha(Z \alpha)^{5}\right)$ [39] and $O\left(\alpha^{2}(Z \alpha)^{4}\right)$ [40]. In both these cases, the LBL scattering was a part of a short-distance correction to the bound electron $g$ factor. Here we focus on the former type of diagrams, where two photons are attached to the electron line.

We start by analyzing the diagram in Fig. 1. The case where both loops are hard was discussed in [40]. In that case, the loops collapse to a point in NRQED, where the diagram is represented by an effective operator with two photon fields. This operator is then matched on the effective spin-dependent potential. Here we consider a situation where only the fermionic loop is hard, while the second loop is soft. This means that only the LBL fermionic loop is a short-distance phenomenon, while photons are part of the long-distance physics. The hard matching leads to the E-H Lagrangian in Eq. (4). The soft loop in the QED diagram is now represented in NRQED by a time-ordered product of the E-H Lagrangian

$$
\frac{1}{3 !} \int d^{4} x \int d^{4} y \int d^{4} z T\left[\mathcal{L}_{\mathrm{I}}(x), \mathcal{L}_{\mathrm{I}}(y), \mathcal{L}_{\mathrm{EH}}(z)\right],
$$

with the interaction Lagrangian containing the leading Coulomb interaction and a Pauli interaction

$$
\mathcal{L}_{\mathrm{I}}=\chi_{e}^{\dagger}\left(-e A_{0}+c_{F} e \frac{\boldsymbol{\sigma} \cdot \boldsymbol{B}}{2 m}\right) \chi_{e} .
$$

Here $c_{F}=1+\frac{\alpha}{2 \pi}+O\left(\alpha^{2}\right)$ and $\sigma$ is a vector composed of Pauli matrices. The NRQED diagram representing the timeordered product (6) is depicted in Fig. 2.

To perform the second matching step, we compute the amplitude for the diagram shown in Fig. 2; it reads

$$
i c_{2} c_{F} \frac{\alpha^{2}}{m^{4}} \frac{Z e^{3}}{32 m} \frac{Q_{i} Q_{j}}{|\boldsymbol{Q}|} \hat{\chi}_{e}^{\dagger} \sigma_{j} \hat{\chi}_{e} B_{i},
$$

with $Q$ representing the momentum transfer between the electron and the nucleus, and the external magnetic field $\boldsymbol{B}$ that carries zero momentum. $\hat{\chi}_{e}$ denotes nonrelativistic electron spinors. 


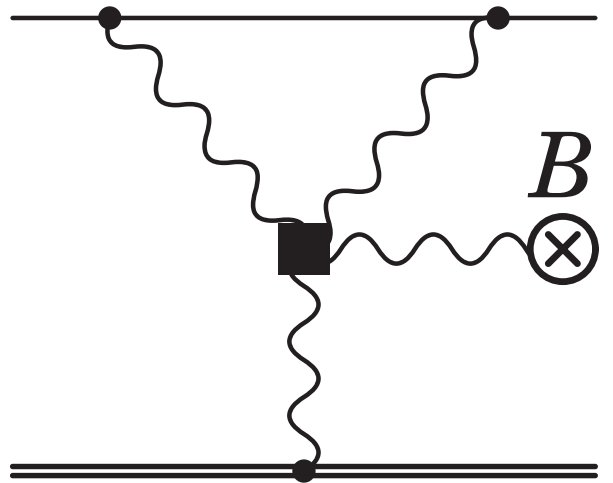

FIG. 2. NRQED diagram corresponding to Fig. 1 with the EulerHeisenberg Lagrangian insertion replacing the electron loop.

We drop the part of the amplitude that does not contribute in $s$ states and, after a Fourier transform, we find the spindependent correction to the PNRQED potential,

$$
e \int d^{3} r\left[\chi_{e}^{\dagger} \frac{\boldsymbol{\sigma} \cdot \boldsymbol{B}_{\mathrm{ext}}}{2 m} \chi_{e}\right](\boldsymbol{x}+\boldsymbol{r}) \delta V(r)\left[N^{\dagger} N\right](\boldsymbol{x}),
$$

with

$$
\delta V(r)=-c_{2} c_{F} \frac{\alpha^{2}}{\pi^{2}} \frac{Z \alpha}{(m r)^{4}} \frac{\pi}{12} .
$$

This potential has $r^{-4}$ dependence and it is thus more singular for small $r$ than the leading Coulomb potential in Eq. (5). Consequently, the matrix element in an $s$ state is divergent and has to be regularized. We use dimensional regularization with space-time dimension $D=4-2 \epsilon$ and find the E-H contribution to the bound electron $g$ factor to be

$$
\Delta g_{\mathrm{EH}}=\left(\frac{\alpha}{\pi}\right)^{2} \frac{28 \pi}{135}(Z \alpha)^{5}\left(\frac{1}{\epsilon}-\ln \frac{(m Z \alpha)^{2}}{\mu^{2}}+\cdots\right),
$$

where dots represent terms that are not logarithmically enhanced. The computation of the matrix element is closely related to the logarithmic correction to the Lamb shift described in [40].

The $1 / \epsilon$ ultraviolet (UV) pole of the matrix element of $\delta V$ cancels with the high-energy contribution shown in Fig. 3. The additional photon connecting the external electron to the nucleus may be understood as a high-energy tail of the electron wave function. This is why only the diagrams related to the
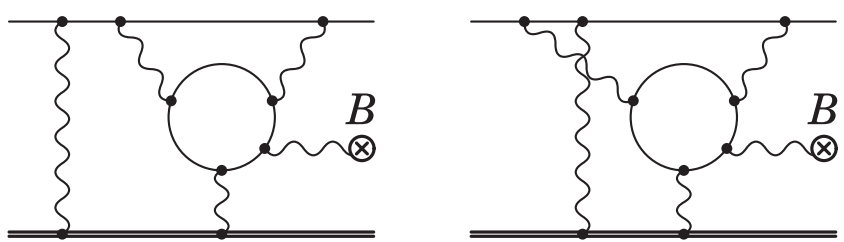

FIG. 3. The high-energy correction to the diagram in Fig. 1 arises from an additional hard photon exchanged between the electron and the nucleus. Two examples are shown. Other diagrams are found by permuting photons coupled to each electron line. The diagram on the right does not contribute to the divergent part in Eq. (13). left diagram in Fig. 3 by permutation of photon attachments to the closed electron loop contribute to the divergent part. In this short distance part of the correction all loop momenta have a hard scaling $(\sim m)$. Reference [41] explains the theory of the high-energy contribution to the bound $g$ factor at $O\left((Z \alpha)^{5}\right)$.

In the short-distance calculation we proceed as in our previous calculations [42,43]. All three-loop integrals are reduced to a small set of master integrals with the so-called Laporta algorithm $[44,45]$ implemented in the program FIRE [46]. Even though we are dealing with diagrams that do not contribute to the Lamb shift, almost all master integrals are the same as before, and their results can be found in [42]. The reason is that master integrals correspond to scalar diagrams, where some of the lines are absent. In most cases, one can transform these master integrals into known ones.

However, there is one new master integral,

$$
\begin{aligned}
& \int \frac{d^{D} k_{1} d^{D} k_{2} d^{D} k_{3} \delta\left(k_{2}^{0}\right)}{k_{1}^{2}\left(k_{1}-k_{2}\right)^{2}\left(k_{3}^{2}+m^{2}\right)\left[\left(k_{2}+k_{3}\right)^{2}+m^{2}\right]} \\
& =-\frac{64 \pi^{7} m^{3}}{3}+O(\epsilon),
\end{aligned}
$$

that could not be checked with previous calculations. For this reason the computation of the hard part alone would not be a sufficient proof of the presence of the logarithm. Fortunately, the hard correction we found,

$$
\Delta g_{\mathrm{H}}=-\left(\frac{\alpha}{\pi}\right)^{2} \frac{28 \pi}{135}(Z \alpha)^{5}\left(\frac{1}{\epsilon}-\ln \frac{m^{2}}{\mu^{2}}+\cdots\right)
$$

is consistent with the soft correction in Eq. (11). Summing Eqs. (11) and (13) we find that $1 / \epsilon$ singularities cancel and obtain our main result,

$$
\Delta g(Z)=\Delta g_{\mathrm{H}}+\Delta g_{\mathrm{EH}}=\left(\frac{\alpha}{\pi}\right)^{2}(Z \alpha)^{5} \frac{28 \pi}{135} \ln \frac{1}{(Z \alpha)^{2}},
$$

from which we read off the coefficient $b_{51}$ in Eq. (3).

It was believed that the missing LBL corrections were small [5]. Our result shows that such estimates have to be carefully scrutinized. Due to the logarithmic enhancement, the correction is larger than anticipated and exceeds other LBL corrections computed previously in [43]. The EFT approach provides a systematic framework for analyzing higher-order corrections, and we advocate a widespread usage of this formalism in future investigations.

For the hydrogenlike carbon ion, currently the best source of the electron mass determination, the resulting relative correction to the $g$ factor and, by the same token, to the electron mass $m$, is

$$
\frac{\Delta g(Z=6)}{g}=\frac{\Delta m}{m}=1.8 \times 10^{-12},
$$

about 17 times smaller than the current experimental error. This correction will likely become important for the measurements in the near future [7]. 
Because of the factor $Z^{5}$, the correction grows rapidly for heavier ions. For the experimentally important silicon [47],

$$
\frac{\Delta g(Z=14)}{g}=0.9 \times 10^{-10},
$$

exceeding the accepted theoretical uncertainty of $0.7 \times 10^{-10}$ [3]. This is likely because Ref. [3] fitted unknown higher-order corrections, assuming a vanishing $b_{51}$, as we explained below Eq. (2).

For the future, two extensions of this work are of interest. While we have determined the E-H effect in a one-electron hydrogenlike ion, few-electron systems, especially lithiumand boronlike ions, are also experimentally relevant [48]. It would also be interesting to evaluate the E-H correction for a muonic atom [49] where it should be further enhanced by the logarithm of the electron to muon mass ratio.

R.S. would like to thank Martin Beneke for useful discussions. The loop diagrams were calculated with FORM [50,51]. This work was supported by the Natural Sciences and Engineering Research Council of Canada and by the Deutsche Forschungsgemeinschaft (DFG, German Research Foundation) under Grant No. 396021762-TRR 257.
[1] T. Aoyama, T. Kinoshita, and M. Nio, Theory of the anomalous magnetic moment of the electron, Atoms 7, 28 (2019).

[2] S. Laporta, High-precision calculation of the 4-loop contribution to the electron $g-2$ in QED, Phys. Lett. B 772, 232 (2017).

[3] J. Zatorski, B. Sikora, S. G. Karshenboim, S. Sturm, F. KöhlerLanges, K. Blaum, C. H. Keitel, and Z. Harman, Extraction of the electron mass from $g$-factor measurements on light hydrogenlike ions, Phys. Rev. A 96, 012502 (2017).

[4] B. Sikora, V. Yerokhin, N. Oreshkina, H. Cakir, C. Keitel, and Z. Harman, Theory of the two-loop self-energy correction to the $g$ factor in nonperturbative Coulomb fields, Phys. Rev. Research 2, 012002 (2020).

[5] V. A. Yerokhin and Z. Harman, Two-loop QED corrections with closed fermion loops for the bound-electron $g$ factor, Phys. Rev. A 88, 042502 (2013).

[6] V. Debierre, B. Sikora, H. Cakir, N. Oreshkina, V. Yerokhin, C. Keitel, and Z. Harman, Two-loop virtual light-bylight scattering corrections to the bound-electron $g$ factor, arXiv:2007.12244.

[7] S. Sturm et al., The ALPHATRAP experiment, Eur. Phys. J.: Spec. Top. 227, 1425 (2019).

[8] V. M. Shabaev, D. A. Glazov, N. S. Oreshkina, A. V. Volotka, G. Plunien, H.-J. Kluge, and W. Quint, $g$-Factor of Heavy Ions: A New Access to the Fine Structure Constant, Phys. Rev. Lett. 96, 253002 (2006).

[9] V. A. Yerokhin, E. Berseneva, Z. Harman, I. I. Tupitsyn, and C. H. Keitel, Weighted difference of $g$ factors of light Li-like and H-like ions for an improved determination of the finestructure constant, Phys. Rev. A 94, 022502 (2016).

[10] R. H. Parker, C. Yu, W. Zhong, B. Estey, and H. Müller, Measurement of the fine-structure constant as a test of the Standard Model, Science 360, 191 (2018).

[11] G. Gabrielse, S. Fayer, T. Myers, and X. Fan, Towards an improved test of the dtandard model's most precise prediction, Atoms 7, 45 (2019).

[12] V. Debierre, C. Keitel, and Z. Harman, Fifth-force search with the bound-electron $g$ factor, Phys. Lett. B 807, 135527 (2020).

[13] V. A. Yerokhin, K. Pachucki, and V. Patkos, Theory of the Lamb shift in hydrogen and light hydrogen-like ions, Anna. Phys. 531, 1800324 (2019).

[14] W. Heisenberg and H. Euler, Consequences of Dirac's theory of positrons, Z. Phys. 98, 714 (1936).

[15] H. Euler and B. Kockel, The scattering of light by light in Dirac's theory, Naturwissenschaften 23, 246 (1935).
[16] V. Weisskopf, The electrodynamics of the vacuum based on the quantum theory of the electron, K. Dan. Vidensk. Selsk., Mat.Fys. Med. 14N6, 1 (1936), reprinted in [52].

[17] A. Ejlli, F. Della Valle, U. Gastaldi, G. Messineo, R. Pengo, G. Ruoso, and G. Zavattini, The PVLAS experiment: A 25 year effort to measure vacuum magnetic birefringence, Phys. Rep. 871, 1 (2020).

[18] S. Z. Akhmadaliev, G. Y. Kezerashvili, S. G. Klimenko, R. N. Lee, V. M. Malyshev, A. L. Maslennikov, A. M. Milov, A. I. Milstein, N. Y. Muchnoi, A. I. Naumenkov et al., Experimental Investigation of High-Energy Photon Splitting in Atomic Fields, Phys. Rev. Lett. 89, 061802 (2002).

[19] R. Lee, A. Maslennikov, A. Milstein, V. Strakhovenko, and Y. Tikhonov, Photon splitting in atomic fields, Phys. Rep. 373, 213 (2003).

[20] A. Milstein and M. Schumacher, Present status of Delbruck scattering, Phys. Rep. 243, 183 (1994).

[21] G. Aad et al. (ATLAS Collaboration), Observation of Light-byLight Scattering in Ultraperipheral $\mathrm{Pb}+\mathrm{Pb}$ Collisions with the ATLAS Detector, Phys. Rev. Lett. 123, 052001 (2019).

[22] F. V. Tkachev, Theory of asymptotic operation. A summary of basic principles, Sov. J. Part. Nucl. 25, 649 (1994), arXiv:hep$\mathrm{ph} / 9701272$.

[23] M. Beneke and V. A. Smirnov, Asymptotic expansion of Feynman integrals near threshold, Nucl. Phys. B 522, 321 (1998).

[24] V. A. Smirnov, Applied asymptotic expansions in momenta and masses, Springer Tracts Mod. Phys. 177, 1 (2002).

[25] A. Czarnecki and V. A. Smirnov, Threshold behavior of Feynman diagrams: The master two-loop propagator, Phys. Lett. B 394, 211 (1997).

[26] A. Czarnecki and K. Melnikov, Two-Loop QCD Corrections to the Heavy Quark Pair Production Cross-Section in $e^{+} e^{-}$Annihilation Near the Threshold, Phys. Rev. Lett. 80, 2531 (1998).

[27] W. E. Caswell and G. P. Lepage, Effective Lagrangians for bound state problems in QED, QCD, and other field theories, Phys. Lett. B 167, 437 (1986).

[28] R. J. Hill, G. Lee, G. Paz, and M. P. Solon, NRQED Lagrangian at order $1 / M^{4}$, Phys. Rev. D 87, 053017 (2013).

[29] A. Pineda and J. Soto, The Lamb shift in dimensional regularization, Phys. Lett. B 420, 391 (1998).

[30] A. Pineda and J. Soto, Potential NRQED: The positronium case, Phys. Rev. D 59, 016005 (1998).

[31] M. Beneke, Perturbative heavy quark-antiquark systems, PoS hf8, 009 (1999). 
[32] R. Szafron, E. Y. Korzinin, V. A. Shelyuto, V. G. Ivanov, and S. G. Karshenboim, Virtual Delbrück scattering and the Lamb shift in light hydrogenlike atoms, Phys. Rev. A 100, 032507 (2019).

[33] U. D. Jentschura, A. Czarnecki, and K. Pachucki, Nonrelativistic QED approach to the Lamb shift, Phys. Rev. A 72, 062102 (2005).

[34] K. Pachucki, U. D. Jentschura, and V. A. Yerokhin, Nonrelativistic QED Approach to the Bound-Electron $g$ Factor, Phys. Rev. Lett. 93, 150401 (2004); Erratum: Nonrelativistic QED Approach to the Bound-Electron g Factor [Phys. Rev. Lett. 93, 150401 (2004)], 94, 229902(E) (2005).

[35] K. Pachucki, A. Czarnecki, U. D. Jentschura, and V. A. Yerokhin, Complete two-loop correction to the bound-electron $g$ factor, Phys. Rev. A 72, 022108 (2005)

[36] K. Pachucki, Long-wavelength quantum electrodynamics, Phys. Rev. A 69, 052502 (2004).

[37] C. Peset and A. Pineda, The Lamb shift in muonic hydrogen and the proton radius from effective field theories, Eur. Phys. J. A 51, 156 (2015).

[38] C. Peset, Effective field theories for muonic hydrogen, EPJ Web Conf. 137, 08013 (2017).

[39] S. G. Karshenboim and A. I. Milstein, Delbruck scattering and the $g$ factor of a bound electron, Phys. Lett. B 549, 321 (2002).

[40] A. Czarnecki and R. Szafron, Light-by-light scattering in the Lamb shift and the bound electron $\mathrm{g}$ factor, Phys. Rev. A 94, 060501(R) (2016).

[41] K. Pachucki and M. Puchalski, One-loop binding corrections to the electron $g$ factor, Phys. Rev. A 96, 032503 (2017).

[42] M. Dowling, J. Mondéjar, J. H. Piclum, and A. Czarnecki, Radiative-nonrecoil corrections of order $\alpha^{2}(Z \alpha)^{5}$ to the Lamb shift, Phys. Rev. A 81, 022509 (2010).
[43] A. Czarnecki, M. Dowling, J. Piclum, and R. Szafron, TwoLoop Binding Corrections to the Electron Gyromagnetic Factor, Phys. Rev. Lett. 120, 043203 (2018).

[44] S. Laporta and E. Remiddi, The analytical value of the electron $(g-2)$ at order $\alpha^{3}$ in QED, Phys. Lett. B 379, 283 (1996).

[45] S. Laporta, High-precision calculation of multi-loop Feynman integrals by difference equations, Int. J. Mod. Phys. A 15, 5087 (2000).

[46] A. V. Smirnov, FIRE5: A C ++ implementation of Feynman Integral REduction, Comput. Phys. Commun. 189, 182 (2014).

[47] S. Sturm, A. Wagner, B. Schabinger, J. Zatorski, Z. Harman, W. Quint, G. Werth, C. H. Keitel, and K. Blaum, g Factor of Hydrogenlike ${ }^{28} \mathrm{Si}^{13+}$, Phys. Rev. Lett. 107, 023002 (2011).

[48] D. Glazov, F. Köhler-Langes, A. V. Volotka, K. Blaum, F. Heiße, G. Plunien, W. Quint, S. Rau, V. M. Shabaev, S. Sturm, and G. Werth, $g$ Factor of Lithiumlike Silicon: New Challenge to Bound-State QED, Phys. Rev. Lett. 123, 173001 (2019).

[49] B. Sikora, H. Cakir, N. Michel, V. Debierre, N. S. Oreshkina, N. A. Belov, V. A. Yerokhin, C. H. Keitel, and Z. Harman, Improving the accuracy of the muon mass and magnetic moment anomaly via the bound-muon $g$ factor, Phys. Rev. D 97, 111301(R) (2018).

[50] B. Ruijl, T. Ueda, and J. Vermaseren, FORM version 4.2, arXiv: 1707.06453

[51] J. A. M. Vermaseren, New features of FORM, arXiv:math$\mathrm{ph} / 0010025$.

[52] J. Schwinger, Selected Papers On Quantum Electrodynamics (Dover, New York, 1958). 